\title{
Review of: "Is delay to radical cystectomy following BCG failure oncologically safe?"
}

\author{
Roberto Carando
}

Potential competing interests: The author(s) declared that no potential competing interests exist.

This review considers various treatments employed in SBTT. At the moment we have some new promising treatment for the BCG failures (e.g. nadofaragene firafenovec) and others (yet evaluated in other studies always involving "BCG failures") with a demonstrated low efficacy. Another issue is the parameter of recurrence innthese patients. Not all the cited studies considered the "recurrence in the sane way as we have to consider the difference among the BCG no reaponders (failures, relapses). Anyway this review focuses on a pivotal and challenging issue: when and how to change the therapeutical approach in "high risk Patients". From this point of view there is at the moment a lack of studies, in particular to evaluate new drugs (also administred through devices) and in particular a lack to approach these studies with agreed and common parameters in terms of recurrence (and strategies to treat e.g. recurrences low grade/low stage, considering the starting risk) and a not joint or common way to approach the timing of follow-up and the way to evaluate micromethastasis in the period of follow-up. Anyway, this could be a short and interesting review that opens the aforementioned questions. 